\title{
En Argentina, la exposición pasiva al humo de tabaco en edificios públicos es la más alta de América Latina
}

\section{Objetivo}

Evaluar la concentración de humo de tabaco ambiental en lugares públicos, en las capitales de Argentina, Brasil, Chile, Costa Rica, Paraguay, Perú y Uruguay.

Diseño

Estudio diagnóstico de corte transversal.

\section{Lugar}

Lugares públicos (aeropuertos, hospitales, escuelas, edificios gubernamentales, restaurantes, bares) de 7 ciudades capitales de 7 países latinoamericanos.

\section{Descripción de los dispositivos}

Dispositivos que utilizan un filtro de metal tratado con bisulfato de sodio.

\section{Métodos}

Se colocaron 683 dispositivos para medir nicotina en fase gaseosa en los distintos lugares públicos seleccionados sin conocer el nivel de consumo de tabaco previo. Se colocaron durante una semana en los bares y restaurantes, y durante dos semanas en los demás lugares públicos. Se analizó la cantidad de nicotina recolectada, usando cromatografía gaseosa con detección selectiva de nitrógeno (cantidad de nicotina recolectada en ug por el volumen de aire del ambiente en m3). Se colocaron aparatos "testigos" en el $10 \%$ de los dispositivos colocados.

\section{Análisis estadístico}

Se reportan medianas y rangos intercuartilos (IQRs) para describir los datos, y box plots en escala logarítmica para presentar los gráficos (por país) por cada tipo de institución. (Ver gráfico).

\section{Medición de Resultado Principal}

Concentración de nicotina ambiental.

\section{Resultados principales}

De los 683 aparatos colocados se analizaron 633. Se detectó nicotina ambiental en la mayoría (94\%) de los lugares evaluados. En 155 de los 163 hospitales evaluados (95\%), se detecto nicotina ambiental. La mediana de concentración fue mayor en Argentina y Uruguay (1.33 [IQR, 0.51-3.12] ug/m3 y 0.8 [IQR, 0.30-1.69] ug/m3, respectivamente), seguido por Chile (0.13 [IQR, 0.03-0.47] ug/m3), y menor en el resto de los países. En las escuelas secundarias, se detecto nicotina ambiental en 73 de 94 muestras (78\%). A nivel de edificios gubernamentales, la mayor concentración se encontró en Argentina y Uruguay. Altas concentraciones fueron halladas en el aeropuerto internacional de Argentina, y en aeropuertos de Chile, Uruguay, y Perú. La mediana en restaurantes fue 1.24 (IQR, 0.412.48) ug/m3; donde la mayor concentración de nicotina se encontró en las áreas para no fumadores $(13.3 \mathrm{ug} / \mathrm{m} 3)$ ubicadas en el segundo piso por arriba del área fumador conectadas por escaleras. Los bares tuvieron las concentraciones más altas (mediana 3.65 [IQR, 1.55-5.12] ug/m3), con pocas diferencias entre los países.

\section{Conclusiones}

Se detectó nicotina ambiental en la mayoría de los lugares estudiados, siendo alta o muy alta en edificios públicos, en especial en escuelas y hospitales donde regularmente esta prohibido fumar. Argentina y Uruguay mostraron la mayor concentración de nicotina en los lugares públicos. Las áreas para no fumadores demostraron, una vez más, no ser efectivas para proteger la salud de los no fumadores.

Fuente de financiamiento: Organización panamericana de la salud e Instituto para el control global del tabaco, Escuela de Salud Pública "Johns Hopkins Bloomberg".

\section{Comentario}

El humo de tabaco ajeno es causa de enfermedad y muerte ${ }^{1}$. Numerosos meta-análisis muestran que es responsable de un 20 $30 \%$ de incremento de riesgo de padecer cáncer de pulmón ${ }^{2}$ y un $30-40 \%$ de infarto de miocardio ${ }^{3}$. El tabaquismo pasivo es responsable de 1 de cada 10 muertes producidas por el tabaco. Estimaciones de la Agencia de Protección del Medio Ambiente (EPA) de USA, demuestran que si todos los ambientes cerrados fueran libres de humo de tabaco (ALH), se evitarían 120.000 a 200.000 muertes por año en todo el continente americano ${ }^{4}$. Las normas internacionales recomiendan un orden de prioridad en el proceso de implementación de ALH, comenzando por el gobierno, las instituciones de salud y de educación ${ }^{5}$. Esta claro que es muy poco probable poder avanzar hacia ALH en bares y restaurantes, si aun se fuma en las escuelas o en los hospitales. En la mayoría de los países desarrollados existe una alta adhesión a los ALH que ya son considerados la "norma social" vigente. El presente estudio es el primero que evalúa la concentración de nicotina ambiental en América Latina y su propósito fue el de describir y cuantificar objetivamente la exposición pasiva al humo de tabaco en nuestras poblaciones. Argentina, Uruguay y Chile presentaron niveles muy elevados de nicotina ambiental, en lugares donde está prohibido fumar, como hospitales, escuelas u oficinas de la administración pública. Es de particular preocupación la elevada exposición a humo de tabaco en hospitales y escuelas de Argentina, mostrando la inmadurez que presenta nuestra sociedad (aun entre los profesionales de la salud) en el conocimiento del daño que genera la exposición pasiva al humo de tabaco. Por otra parte, este estudio corrobora una vez más, que separar áreas para fumadores y no fumadores es ineficaz para proteger la salud de las personas. Si bien el estudio presenta algunas limitaciones como la selección por conveniencia de los lugares a estudiar, el escaso número de dispositivos colocados (por razones de factibilidad), no haber tenido en cuenta las características de los edificios en cuanto a tamaño de los ambientes, ventilación, etc, representa un fuerte precedente para los países de nuestra región, aportando una evidencia esencial para la protección de los fumadores pasivos. Sin duda, evidencias de esta naturaleza, favorecerán a la sensibilización para el cambio tanto de la comunidad en general como de los decisores políticos en materia de salud y legislación que promuevan a la implementación de ambientes libres de humo de tabaco.

Gráfico: Concentraciones de nicotina gaseosa en lugares públicos de capitales de América latina

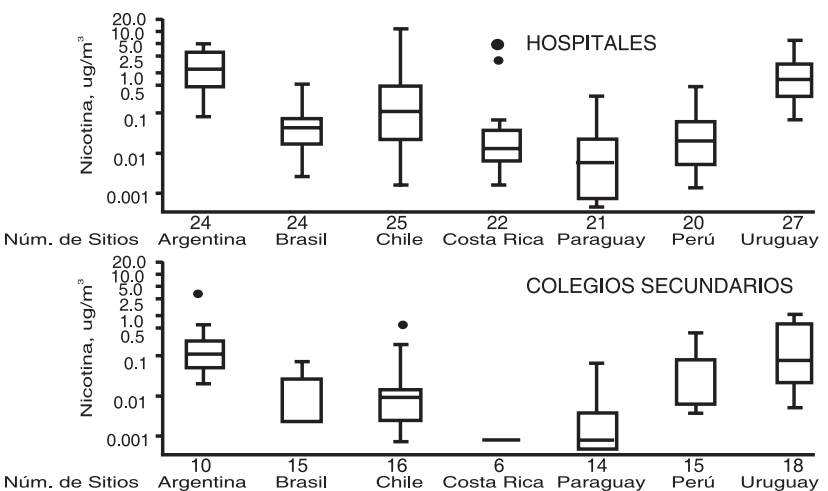

Tomas Smith, Verónica Schoj [ Unidad de Medicina Familiar y Preventiva .Hospital Italiano de Buenos Aires. ]

Smith, T. Schoj V. En Argentina, la exposición pasiva al humo de tabaco en edificios públicos es la mas alta de América Latina. Evid.Actual.Pract.ambul. 2005: 8:6. Comentado de: Secondhand Tobacco Smoke in Public Places in Latin America, 2002-2003. Navaz-Acien A, Peruga A, Breysse P y col. JAMA. 2004, June 9; Vol. 291, (№ 22):2741-5. PMID 15187056. Referencias

1. US Department of Health and Human Services. The health consequences of involuntary smoking: a report of the Surgeon General. Washington DC: US Government Printing Office, 1986. (DHSS Pub No. (PHS) 87-8398) 2. A K Hackshaw, M R Law, N J Wald, The accumulated evidence on lung cancer and environmental tobacco smoke BMJ 1997;315:980-988

3. Law M R, Morris J K, Wald N J. Environmental tobacco smoke exposure and ischaemic heart disease: an evaluation of the evidence. BMJ 1997; 315: 973-80.

4. EPA. Respiratory health effects of passive smoking: lung cancers and other disorders: US Environmental Protection Agency. Washington: Office of Air and Radiation, 1992. (EPA/600/6-90/006F).

5. CDC Taking actions on second hand smoke. http://www.cdc.gov/tobacco/ETS_Toolkit/index.htm. 